\title{
AIRBORNE 2-MICRON DOUBLE PULSED DIRECT DETECTION IPDA LIDAR FOR ATMOSPHERIC $\mathrm{CO}_{2}$ MEASUREMENT
}

\author{
Jirong Yu ${ }^{1}$, Mulugeta Petros ${ }^{1}$, Tamer Refaat ${ }^{1}$, Karl Reithmaier ${ }^{2}$, Ruben Remus ${ }^{1}$, Upendra Singh ${ }^{1}$, \\ Will Johnson ${ }^{3}$, Charlie Boyer ${ }^{1}$, James Fay ${ }^{1}$, Susan Johnston ${ }^{2}$, Luke Murchison ${ }^{1}$ \\ ${ }^{1}$ NASA Langley Research Center, MS 468, Hampton, VA 23681 USA, j.yu@nasa.gov \\ ${ }^{2}$ Science System \&Applications, Inc, One Enterprise Parkway, Hampton, Virginia 23666 USA \\ ${ }^{3}$ Michigan Aerospace Corporation, 1777 Highland Dr. \# B, Ann Arbor, MI 48108 USA
}

\begin{abstract}
An airborne 2-micron double-pulsed Integrated Path Differential Absorption (IPDA) lidar has been developed for atmospheric $\mathrm{CO}_{2}$ measurements. This new instrument has been flown in spring of 2014 for a total of ten flights with 27 flight hours. This IPDA lidar provides high precision measurement capability by unambiguously eliminating contamination from aerosols and clouds that can bias the results.
\end{abstract}

\section{INTRODUCTION}

Active sensing of carbon dioxide concentrations will significantly increase the understanding of the gas sources, sinks, and fluxes worldwide. The mid-IR wavelength regions at $1.57 \mu \mathrm{m}$ and $2.05 \mu \mathrm{m}$ are considered suitable for atmospheric $\mathrm{CO}_{2}$ measurements due to the existence of distinct absorption features for the gas at these particular wavelengths. NASA Langley Research Center (LaRC) developed a new approach to use double pulsed, high energy 2-micron IPDA lidar instrument to measure atmospheric $\mathrm{CO}_{2}$. The pulsed lidar approach inherently provides means for determining range across the scattering targets. The reflected signals can be resolved between aerosols, clouds, and topographical surfaces. This lidar is operated on the long wavelength wing of $\mathrm{CO}_{2} \mathrm{R}(30)$ absorption line at $2050.967 \mathrm{~nm}$ $\left(4875.749 \mathrm{~cm}^{-1}\right)$ in the side-line operation mode. The $\mathrm{R}(30)$ line is an excellent absorption line for the measurements of $\mathrm{CO}_{2}$ in $2 \mu \mathrm{m}$ wavelength region with regard to the strength of the absorption lines, low susceptibility to atmospheric temperature variability, and freedom from problematic interference with other absorption lines [1]. This paper describes the development of a 2-micron pulsed IPDA lidar instrument that measures atmospheric $\mathrm{CO}_{2}$ concentration between ground and airborne platform.

\section{IPDA LIDAR SYSTEM}

High-precision and accurate atmospheric $\mathrm{CO}_{2}$ measurements impose stringent requirements on the lidar transmitter and receiver parameters, such as laser energy, pulse repetition rate, laser frequency control accuracy, telescope design and aperture size, high sensitivity with low noise detector and receiver design [2]. Figure 1 depicts the block diagram for the 2-micron pulsed IPDA instrument. It contains laser transmitter, thermal control unit for laser transmitter, wavelength tuning, locking and switching unit, telescope, aft optics and detector unit, signal preamplifiers, data acquisition unit, computer control and signal process unit, and data display.

The compact, rugged, highly reliable laser transmitter is based on the Ho:Tm:YLF highenergy 2-micron pulsed laser technology [3]. The laser transmitter is designed to be operated in a unique double pulse format that can produce two pulse pair in $10 \mathrm{~Hz}$ operation. Typically, the output energies of the laser transmitter are $100 \mathrm{~mJ}$ and $45 \mathrm{~mJ}$ for the first pulse and the second pulse, respectively. The first pulse is injection seeded with on-line frequency and the second pulse with off-line frequency. The double pulse operation is a unique feature of this Holmium (Ho) and Thulium $(\mathrm{Tm})$ co-doped laser that has several advantages. First, the laser can be more efficient. It provides two Q-switched pulses with a single pump pulse. Second, since the time interval between the first and second pulses is typically about $150-200 \mu$ s, the foot print overlap on the ground between the two pulses is greater than $95 \%$ for an airborne platform. This mitigates the effect of the surface reflection difference between the on-and-off line pulses on the precision of the $\mathrm{CO}_{2}$ column density measurements. Third, the pulse pair is from the same laser. No beam combining or additional beam control is needed. The laser transmitter is 
$11.5 \times 26.5 \times 6.4$ inch $(29 \times 67.3 \times 16.5 \mathrm{~cm})$ in size, and weighted less than 60lbs.

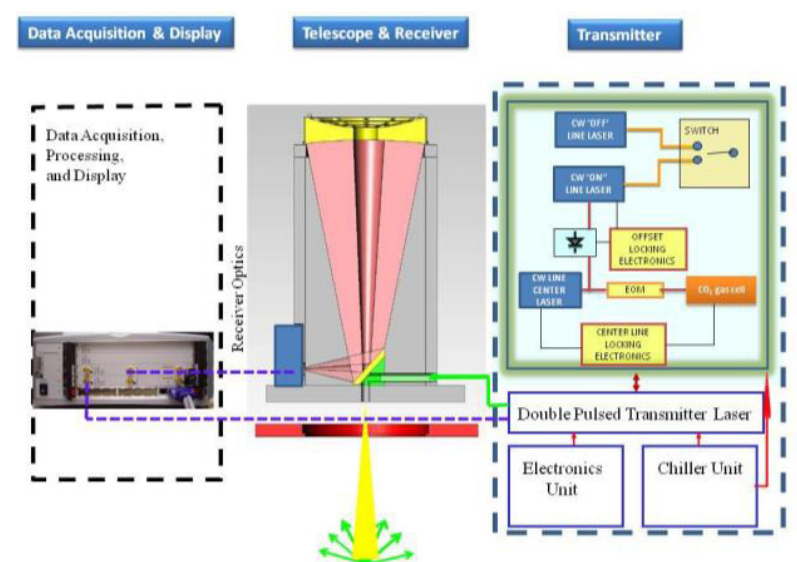

Fig. 1. 2-micron $\mathrm{CO}_{2}$ IPDA instrument block diagram

The exact wavelengths of the pulsed laser transmitter are controlled by the wavelength control unit. The first pulse and the second pulse are injection seeded alternately by the on-line frequency and the off-line frequency. To obtain the wavelength accuracy and stability, a master wavelength reference against a sample of $\mathrm{CO}_{2}$ in a gas cell is established. One of the CW lasers, called the center-line reference, is locked on the center of the $\mathrm{CO}_{2}$ absorption line $\mathrm{R}(30)$ by a frequency modulation spectroscopic technique [4]. A second laser, called the tunable side-line, is referenced to the center-line laser by a heterodyne technique. By monitoring the heterodyne beat signal between the two lasers, the amount of detuning from the $\mathrm{R}(30)$ center can be determined and locked. Typically, it is locked at $2-4 \mathrm{GHz}$ from the peak of R30 absorption line. A third CW laser provides the off-line wavelength. It is locked to a high precision wavemeter. The wavelength locking accuracy is less than $2 \mathrm{MHz}$ and $30 \mathrm{MHz}$ for the on-line and off-line frequencies, respectively. The three $\mathrm{CW}$ lasers, an EO modulator, two detector units, an optical switch, fiber couplers and connectors are all packaged in the custom designed 3U 19 inch rack mountable box.

The telescope is a custom designed Newtonian type with $40 \mathrm{~cm}$ diameter primary mirror size. This primary mirror is made of aluminum with diamond turning machining technique. The telescope is designed to maintain the focus point position in the temperature range between 5 and $35{ }^{\circ} \mathrm{C}$. The lidar return signal is split into two channels, a high gain channel with $90 \%$ of signal power and a low gain channel with $10 \%$ of the signal power. A Hamamatsu InGaAs PIN photodiodes, model G5853-203, is selected and characterized for the airborne IPDA lidar application. This detector has two stage of TEC (thermo-electric cooler) and can be cooled down to $-20^{\circ} \mathrm{C}$. To obtain fast response and low noise required for the lidar signal detection, the diameter of the detector active area is limited to 300 micron. Thus, the NEP value is specified at $2 \times 10^{-13} \mathrm{~W} / \mathrm{Hz}^{0.5}$ at $-20^{\circ} \mathrm{C}$, which is suited for the airborne IPDA lidar.

The data acquisition unit is based on two digitizers. The first is a 10-Bit, $2 \mathrm{GS} / \mathrm{s}$ digitizer (Agilent; U1065A) for laser energy monitoring and the second is a 12-bit, $420 \mathrm{MS} / \mathrm{s}$ digitizer (Agilent; U1066A) for measuring the hard target returns. Detectors are coupled to the digitizers through variable gain, high speed trans-impedance amplifiers (FEMTO; DHPCA-100). Digitizers and data storage are hosted through a personal computer that runs Microsoft XP with a 64-bit/66 $\mathrm{MHz}$ PCI bus. The system is capable of transferring data at sustained rates up to $400 \mathrm{MB} / \mathrm{s}$ to the host computer.

A simple real time data processing algorithm is implemented to show the lidar returning signal quality. It displays the laser energy monitor signal at on-and-off line wavelengths, the raw data from the ground returning signal, the range information from the lidar return, and the first order estimate of the Differential Absorption Optical Depth (DAOD). These signals aids to verify correct lidar operation.

\section{AIRBORNE DEMONSTRATION}

After the IPDA lidar has been integrated in class 10000 clean room environment, it was installed inside a mobile trailer for ground testing using calibrated hard targets before aircraft integration. Five calibrated targets with different reflections at 2-micron wavelength were set up 857 meters away from the lidar trailer. Differential optical depth measurements were obtained by calculating the natural logarithm of the off-line to the on-line return energy after normalization to the transmitted laser energy (laser energy monitor). 
The theoretical differential optical depth was derived using CAPABLE meteorological data and $\mathrm{CO}_{2}$ in-situ gas analyzer (LiCor; LI-840A) and US standard model [5]. In addition, the IPDA measured differential optical depth was converted to $\mathrm{CO}_{2}$ dry mixing ratio, and was compared to the in-situ gas analyzer at the site. General agreement of the $\mathrm{CO}_{2}$ temporal profiles were observed between these two measurements, verifying the 2$\mu \mathrm{m}$ IPDA lidar operation.

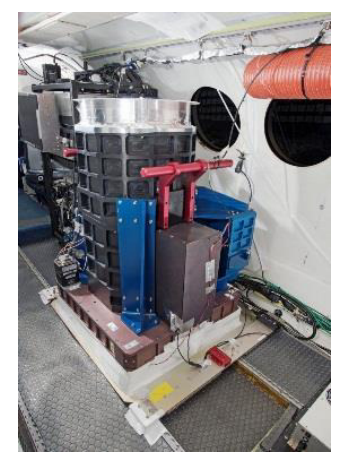

Fig. 2. The double-pulsed 2- $\mu \mathrm{m} \mathrm{CO} 2$ IPDA lidar integrated instrument inside the NASA B-200 research aircraft

The double-pulsed 2- $\mu \mathrm{m} \mathrm{CO}_{2}$ IPDA lidar is designed for integration into a small research aircraft. The IPDA instrument size, weight and power consumption were restricted to the NASA B-200 payload requirements. This allows the system to be easily adopted in any larger airborne research platform, such as the NASA DC-8 aircraft, for future missions. In addition to the IPDA lidar, other housekeeping instruments were integrated into the B-200 aircraft. These included the $\mathrm{CO}_{2}$ in-situ sensor for dry mixing ratio measurement, GPS for aircraft position, altitude and angles measurements and video recorder for target identification. Besides, aircraft built-in sensors provided altitude, pressure, temperature and relative humidity sampling at the flight position. Time stamps were adjusted to the GPS global timing. Figure 2 shows the integrated IPDA instrument inside the NASA B-200 aircraft.

The airborne IPDA lidar instrument measures the total integrated column content of $\mathrm{CO}_{2}$ from the instrument to the ground but with weighting that can be tuned by controlling the transmitted on-line wavelengths. Therefore, the transmitter could be tuned to weight the column measurement to the surface for optimum $\mathrm{CO}_{2}$ sources and sinks interaction studies or up to the free troposphere for optimum transport studies. In fact, it is one of the advantages that the 2-micron pulsed IPDA instrument can provide.

The 2- $\mu \mathrm{m} \mathrm{CO}_{2}$ IPDA lidar airborne testing was conducted during ten daytime flights, spanning more than 27 hours, during March 20, 2014 through April 10, 2014. Meteorological balloon radiosonde was independently lunched from LaRC site, mostly during the beginning and the end of each flight. This allows for atmospheric pressure, temperature and relative humidity vertical profiling estimates for IPDA modelling verifications. IPDA lidar airborne testing included different operating and environmental conditions. Environmental conditions included different flight altitude up to $8.3 \mathrm{~km}$, different ground target conditions such as vegetation, soil, ocean, snow and sand and different cloud conditions. Two flights targeted power plant incinerators for investigating the IPDA sensitivity to $\mathrm{CO}_{2}$ plums. Table 1 lists these flights and summarizes the conditions. The lidar instrument is robust during all of the flights. In spite of the mechanical stresses due to shaking and vibration in the small aircraft environment, the IPDA lidar did not lose alignment resulting in about $190 \mathrm{~GB}$ worth of raw data.

TABLE 1. Summary of the 2-micron $\mathrm{CO}_{2}$ IPDA lidar flights during March 20, 2014 to April 10, 2014 on board the NASA B200 research aircraft

\begin{tabular}{|c|l|l|l|l|}
\hline $\begin{array}{c}\text { Flig } \\
\text { ht \# }\end{array}$ & Date & \multicolumn{2}{|c|}{$\begin{array}{c}\text { Local Time } \\
\text { Start }\end{array}$} & Objective \\
\hline 1 & $3 / 20$ & $1: 50 \mathrm{p}$ & $3: 42 \mathrm{p}$ & Engineering \\
\hline 2 & $3 / 21$ & $2: 17 \mathrm{p}$ & $5: 00 \mathrm{p}$ & Engineering \\
\hline 3 & $3 / 24$ & $8: 50 \mathrm{a}$ & $11: 50 \mathrm{a}$ & Engineering \\
\hline 4 & $3 / 27$ & $7: 15 \mathrm{a}$ & $10: 15 \mathrm{a}$ & Surface \\
\hline 5 & $3 / 27$ & $1: 35 \mathrm{p}$ & $4: 00 \mathrm{p}$ & Surface \\
\hline 6 & $3 / 31$ & $1: 30 \mathrm{p}$ & $4: 00 \mathrm{p}$ & Ocean/cloud \\
\hline 7 & $4 / 02$ & $9: 24 \mathrm{a}$ & $11: 47 \mathrm{a}$ & Power station \\
\hline 8 & $4 / 05$ & $1: 10 \mathrm{p}$ & $4: 52 \mathrm{p}$ & With NOAA \\
\hline 9 & $4 / 06$ & $2: 10 \mathrm{p}$ & $5: 10 \mathrm{p}$ & Power Plant \\
\hline 10 & $4 / 10$ & $3: 40 \mathrm{p}$ & $5: 58 \mathrm{p}$ & In-situ \\
\hline
\end{tabular}

On April 5, 2014, the NASA B-200 eighth flight coincided with another NOAA air sampling flight. Due to flight control restrictions, there was a 30 minute time lag between NOAA and NASA 
flights. Nevertheless, the IPDA lidar on board NASA flight sampled the same geographical location as the NOAA flask samples over the Atlantic Ocean out of the east shore of New Jersey. $\mathrm{CO}_{2}$ flask-sampling results and meteorological data provided by NOAA were valuable for the NASA LaRC IPDA lidar instrument testing. Preliminary analysis of the airborne data indicated the IPDA lidar sensitivity to atmospheric $\mathrm{CO}_{2}$ up to $6.6 \mathrm{~km}$ altitude over ocean target. Figure 3 compares the $\mathrm{CO}_{2}$ differential optical depth obtained from the IPDA data and modelled from NOAA flask sampling data. IPDA indicated a consistent $\mathrm{CO}_{2}$ differential optical depth offset of about 0.07.

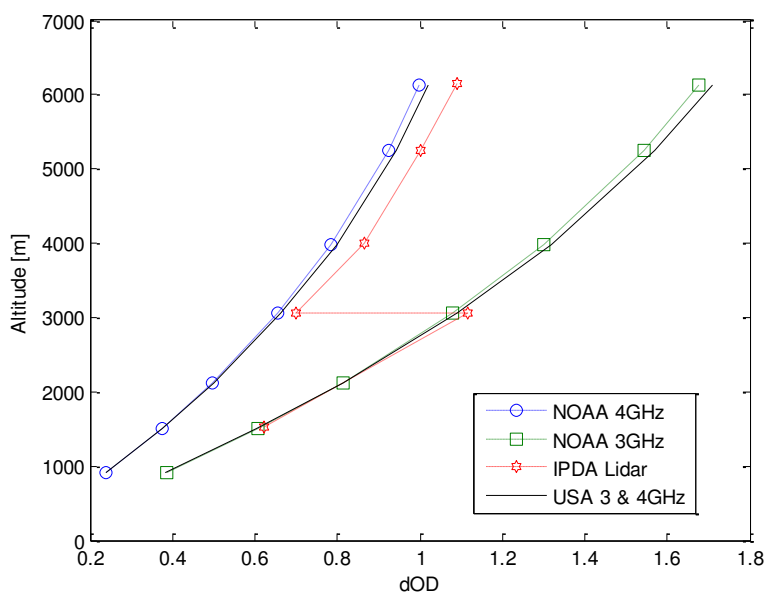

Fig. 3. $\mathrm{CO}_{2}$ differential optical depth versus altitude calculated from NOAA flask sample data and from the IPDA lidar. Results are compared to US standard model calculations

\section{CONCLUSIONS}

NASA LaRC developed a double-pulse, 2- $\mu \mathrm{m}$ integrated path differential absorption (IPDA) lidar instrument for atmospheric $\mathrm{CO}_{2}$ measurement. Advantages of the 2-micron high energy pulsed IPDA remote sensing technique include high signal-to-noise ratio measurement with accurate ranging; favorable weighting function towards ground surface to measure the source and sinks of the $\mathrm{CO}_{2}$; and the capability to directly eliminate contaminations from aerosols and clouds to yield high accuracy $\mathrm{CO}_{2}$ column measurements. The IPDA lidar structure is compactly and ruggedly packaged to fit in the NASA B-200 research aircraft. Ground and airborne testing of the $2-\mu \mathrm{m}$ IPDA lidar was conducted at NASA LaRC through several validation procedures. This included instrument performance modeling through standard atmosphere and meteorological sampling. IPDA $\mathrm{CO}_{2}$ differential optical depth measurement results agree with ground in-situ measurements and with $\mathrm{CO}_{2}$ airborne sampling conducted by NOAA. Further detailed data processing is under work.

\section{ACKNOWLEDGEMENT}

The authors would like to thank NASA Earth Science Technology Office for funding this project. The authors acknowledge the supports from the Engineering and Research Services Directorates at NASA Langley Research Center. Thanks are also due to the dedicated efforts of the Research Systems Integration Branch personnel who made airborne flight testing possible. Acknowledgements are also due to the LaRC CAPABLE team and NOAA for providing public information that is significant for the science validation process. The authors also would like to thank Dr. Robert Menzies at JPL, Dr. Syed Ismail and Tony Notari at LaRC for very useful discussion, advice and help.

\section{REFERENCES}

[1] Ambrico, P. E., A. A. Amodeo, P. D. Gilaramo, and N. Spinelli, 2000: Sensitivity analysis of differential absorption lidar measurements in the midinfrared region, Appl. Opt., 39, 6847-6865

[2] Ehret G., C. Kiemle, 2005: Requirement definition for future DIAL instruments, final report to ESA contract, p64

[3] Yu J., U. N. Singh, N. P. Barnes, and M. Petros, 1998: 125-mJ diode-pumped injection-seeded Ho:Tm:YLF laser, Optics Letters 23, 780-782

[4] Koch, G. J., J. Y. Beyon, F. Gibert, B. W. Barnes, S. Ismail, M. Petros, P. J. Petzar, J. Yu, E. A. Modlin, K. J. Davis, and U. N. Singh, 2008: "Side-line tunable laser transmitter for Differential Absorption Lidar measurement of $\mathrm{CO}_{2}$ : Design and application to atmospheric measurement", Appl. Opt. 47, 944-956

[5] G. Anderson, S. Clough, F. Kneizys, J. Chetwynd, and E. Shettle, 1986: AFGL atmospheric constituent profiles (0-120km), Air Force Geophysics Laboratory, AFGL-TR-86-0110, 954 